\title{
MOLECULAR DYNAMICS CALCULATIONS OF THE THERMAL CONDUCTIVITY OF SILICA BASED CRYSTALS
}

\author{
A. J. H. McGaughey and M. Kaviany \\ Department of Mechanical Engineering, University of Michigan \\ Ann Arbor, MI 48109-2125
}

\begin{abstract}
The thermal conductivities of quartz and the siliceous zeolites sodalite, faujasite and zeolite-A have been calculated using molecular dynamics simulations. The predicted thermal conductivities range over one order of magnitude at room temperature, and the quartz values are in reasonable agreement with the available experimental data. Evidence of phonon scattering is found by considering the lattice statics and dynamics. The lattice pores in zeolites are found to distort the $\mathrm{SiO}_{4}$ tetrahedra, and decrease the thermal conductivity. The integration of the heat current autocorrelation function in the calculation of the thermal conductivity is done in frequency space, allowing the contributions of different vibrational bands to be identified.
\end{abstract}

\section{INTRODUCTION}

Advances in nanoscale technology have led to the possibility of designing and synthesizing materials at the atomic level. Molecular dynamics (MD) simulations allow for the prediction of the properties of potential materials, and for optimization of the design before synthesis is attempted.

Many applications require low thermal conductivity materials. While the thermal conductivity of a solid can be reduced by introducing porosity ${ }^{1,2}$, this also reduces the strength of the material. Amorphous materials also have low thermal conductivities, but in some cases it may be necessary to use a crystalline solid.

The wide range of structures that can be built from the $\mathrm{SiO}_{4}$ tetrahedra present an opportunity to examine how the atomic level assembly of a solid can lead to a wide range of thermal transport properties. To do this, MD simulations have been used to analyze the thermal transport in quartz and siliceous zeolites. After reviewing the basic theory of thermal transport in dielectrics, and examining the analytic and numerical methods available for predicting their thermal conductivities, the results of such simulations are presented. The thermal conductivity trends are explained both qualitatively and quantitatively. Evidence of phonon scattering and localization is found through analysis of the bond lengths and angles, and the vibrational spectra of the structures. Suggestions for the design of new, low thermal conductivity materials are presented.

\section{$\underline{\text { BACKGROUND }}$}

\section{Nanoscale Thermal Transport}

Heat is transferred in dielectric solids through the transport of phonons, quanta of energy associated with lattice vibrations. A harmonic crystal infinite in extent will have an infinite thermal conductivity, as there are no mechanisms through which phonons will interact (i.e., scatter). The thermal conductivity can be reduced through the introduction of phonon scattering centers, which may be impurities, grain boundaries or local distortions of the lattice. Phonon scattering also occurs in three phonon interactions, which result from anharmonicities in the interatomic potential. In solid conductors (metals), electrons also figure into the thermal transport. In the current work, we focus on the phonon (lattice) contribution to the thermal conductivity.

From the gas kinetic theory, the thermal conductivity $k$ is predicted to be ${ }^{3}$

$$
k=\frac{1}{3} C u \lambda,
$$

where $C$ is the volumetric specific heat, $u$ is the mean phonon speed and $\lambda$ is the phonon mean free path. It is from this phenomenological model that much of the understanding of phonon thermal conductivity has been derived.

\section{AnAlytical Predictions of} Thermal Conductivity

The atomic level analytic techniques available for predicting the phonon thermal conductivity can be grouped into two types: those for ordered crystals, and those for disordered crystals and amorphous solids. The general approach for both techniques will be presented.

In a crystalline solid, the phonon transport is phenomenologically represented using a relaxation time, $\tau$, which is related to the phonon mean free

1

American Institute of Aeronautics and Astronautics 
path and speed through

$$
\tau=\frac{\lambda}{u} .
$$

Different scattering mechanisms (e.g., at defects, at grain boundaries, three phonon interactions) are assigned relaxation times that may depend on frequency, temperature and/or lattice parameters. Starting from Eq. (1), and using the Debye model for the specific heat, the temperature dependence of the thermal conductivity is predicted to be $^{3}$

$$
k=\frac{1}{3} u^{2} \int_{0}^{T_{\mathrm{D}} / T} \tau(x) C(x) d x
$$

where

$$
C(x)=\frac{3 k_{\mathrm{B}}}{2 \pi^{2} u^{3}}\left(\frac{k_{\mathrm{B}}}{\hbar}\right)^{3} T^{3} \frac{x^{4} e^{x}}{\left(e^{x}-1\right)^{2}}
$$

and

$$
x=\frac{\hbar \omega}{k_{\mathrm{B}} T} .
$$

In these equations, $T$ is temperature, $T_{\mathrm{D}}$ is the Debye temperature, $k_{\mathrm{B}}$ is the Boltzmann constant, $\hbar$ is the Planck constant divided by $2 \pi$ and $\omega$ is angular frequency. The function $\tau(x)$ is an effective relaxation time at a given frequency. The nature of the different scattering mechanisms determines how they contribute to $\tau(x)^{3}$. Use of this model requires the specification of many parameters associated with the relaxation times, many of which must be fit from experimental data. This relaxation time method has been used to model the thermal conductivities of dielectrics such as germanium ${ }^{4}$, silicon $^{5}$ and alumina ${ }^{6}$.

Cahill and $\mathrm{Pohl}^{7,8}$ have proposed a model for the thermal conductivity of disordered crystals and amorphous solids, given by

$$
\begin{array}{r}
k_{\min }=\left(\frac{\pi}{6}\right)^{1 / 3} k_{\mathrm{B}} n^{2 / 3} \sum_{i}\left[u_{i}\left(\frac{T}{T_{i}}\right)^{2}\right. \\
\left.\int_{0}^{T_{i} / T} \frac{x^{3} e^{x}}{\left(e^{x}-1\right)^{2}} d x\right],
\end{array}
$$

where $T_{i}$ is defined as $u_{i}\left(\hbar / k_{\mathrm{B}}\right)\left(6 \pi^{2} n\right)^{1 / 3}, n$ is the number density of atoms, and the summation is over the three sound modes of the solid. This model is an extension of the Einstein thermal conductivity ${ }^{9}$. The derivation also starts from Eq. (1) and uses a Debye model for the specific heat. Due to the disordered structure, lattice waves do not propagate and the thermal transport is localized. The phonon mean free path is determined based on an assumption of random walks of energy between an atom and a limited number of its nearest neighbors. The predictions of the randon-walk model have been interpreted as a high phonon scattering limit, or a minimum thermal conductivity ${ }^{10}$. Experimental measurements of a range of disordered crystals and amorphous solids have not yielded any thermal conductivities significantly below $k_{\min }{ }^{8}$. In the high temperature limit, the value of $k_{\min }$ becomes constant, i.e.,

$$
\lim _{T \rightarrow \infty} k_{\min }=\left(\frac{\pi}{6}\right)^{1 / 3} \frac{k_{\mathrm{B}} n^{2 / 3}}{2} \sum_{i} u_{i} .
$$

For amorphous silica, $k_{\min }$ reaches $92 \%$ of this value at a temperature of $400 \mathrm{~K}$.

\section{Molecular Simulations Predictions of Thermal Conductivity}

Molecular simulations can be used to predict phonon thermal conductivity using equilibrium or non-equilibrium methods. While some of the techniques have been available for a number of decades, it has not been until the last ten years that computers have become powerful enough to run simulations using realistic potentials over sufficient time scales.

In the equilibrium approach, a Green-Kubo formalism is used to predict that the thermal conductivity will be given by ${ }^{11}$

$$
k=\frac{1}{3 k_{\mathrm{B}} V T^{2}} \int_{0}^{\infty}\langle\mathbf{q}(t) \cdot \mathbf{q}(0)\rangle d t,
$$

where $V$ is the volume of the simulation cell, $t$ is time and $\mathbf{q}$ is the heat current, defined as

$$
\mathbf{q}=\frac{d}{d t} \sum_{i} \mathbf{r}_{i} E_{i}
$$

In Eq. (9), the summation is over the $i$ particles in the system, $\mathbf{r}$ is the location of a particle and $E$ is its total energy (kinetic and potential). For computational ease, Eq.(9) can be recast as

$$
\mathbf{q}=\sum_{i} E_{i} \mathbf{v}_{i}+\frac{1}{2} \sum_{i, j}\left(\mathbf{F}_{i j} \cdot \mathbf{v}_{i}\right) \mathbf{r}_{i j}
$$

where $\mathbf{v}$ is the velocity of a particle and $\mathbf{r}_{i j}$ and $\mathbf{F}_{i j}$ are the distance and force between particles $i$ and $j$. The first term in Eq. (10) corresponds to the contributions of convection, and the second term corresponds to conduction. The integrand of Eq. $(8),\langle\mathbf{q}(t) \cdot \mathbf{q}(0)\rangle$, is the heat current autocorrelation function ( $\mathrm{HCACF}$ ). A large number of time origins within the ensemble average are required for good 
convergence. As well, for a given set of independent variables (e.g., mass, volume and energy), multiple runs are required with different initial conditions to get a proper sampling of phase space ${ }^{12}$.

The heat current is a vector that indicates the magnitude and direction of the flow of heat in a system. At equilibrium, the heat current will oscillate, and over time average to zero. The Green-Kubo approach relates the thermal conductivity to how long it takes for these oscillations to uncorrelate. Previous work using this approach has calculated the thermal conductivity of materials such as $\beta$-silicon carbide $^{12}$, diamond ${ }^{13}$, amorphous silica ${ }^{14} \operatorname{argon}^{15}$, and Lennard-Jones nanofluids ${ }^{16}$.

Equation (8) can also be recast as ${ }^{17}$

$$
k=\left.\frac{1}{3 k_{\mathrm{B}} V T^{2}}[Q(\omega)]\right|_{\omega=0},
$$

where $Q(\omega)$ is the Fourier transform of the heat current. This approach has been used to calculate the conductivity of amorphous ${ }^{17}$ and crystalline ${ }^{18}$ silicon. Non-equilibrium techniques have also been developed $^{19-25}$.

The agreement between the MD thermal conductivity predictions and experimental results varies. The main source of discrepancy is the interatomic potential used. Many potential functions have been developed using elastic properties, with no consideration to thermal transport. For example, the LennardJones potential, widely used in the modelling of Nobel gases, underpredicts the thermal conductivity of Argon by a factor of two ${ }^{15}$. In some $\operatorname{cases}^{12,17,18}$, the use of quantum corrections has been found to improve predictions at low temperatures.

\section{Molecular Simulations}

\section{SilicA STRUCTURES}

Silica structures are constructed from $\mathrm{SiO}_{4}$ tetrahedra. Under ambient conditions, quartz and amorphous silica can be thought of as upper and lower bounds to the thermal conductivity of silica structures. The quartz structure has a high degree of order, while the amorphous material is completely disordered.

Other materials constructed from the $\mathrm{SiO}_{4}$ tetrahedron are found naturally and can be synthesized. Among these are the zeolites ${ }^{26}$, a class of crystal characterized by Angstrom sized pores and channels. One length scale up from the $\mathrm{SiO}_{4}$ tetrahedra, zeolites can be described by secondary building units (SBUs). In Figs. 1 and 2, a number of SBUs are shown in the zeolites sodalite, faujasite and zeolite-A. The SBUs are named based on their geometry. For example, a $6 \mathrm{R} \mathrm{SBU}$ is a ring structure made from 6 oxygen atoms and 6 silicon atoms. Zeolites are used in industrial applications as desiccants, molecular sieves, and catalysts. Typically, the zeolite framework also contains aluminum atoms in place of some of the silicon atoms, and the structure is filled with non-framework anions and diffusing species such as water. In the present simulations, the focus will be on all silicon-oxygen frameworks, also known as siliceous zeolites. Initial coordinates for the structures are taken from $\mathrm{Wycoff}^{27}$.

The four crystals studied using MD are $\alpha$-quartz (Q) and the zeolites sodalite (SOD), faujasite (FAU) and zeolite-A (LTA). The structures are shown in Figs. 1 and 2. All three zeolites are constructed from sodalite cages, which can be thought of as a structure one length scale above the SBUs. In SOD, the sodalite cages are directly joined at the $4 \mathrm{R}$ rings to form a cubic arrangement of cages. In FAU, the sodalite cages are joined by oxygen bridges at the $6 \mathrm{R}$ rings, resulting in a diamond arrangement of cages. LTA is a cubic arrangement of sodalite cages joined by oxygen bridges at the $4 \mathrm{R}$ rings. The complex zeolite structures lead to the existence of distinct oxygen positions in the crystal lattice. These are indicated in Fig. 2 for LTA.

\section{INTERATOMIC POTENTIAL}

The atomic interactions are modeled with the BKS potential $^{28,29}$, where the potential energy $U_{i j}$ between atoms $i$ and $j$ is given by

$$
U_{i j}=\frac{q_{i} q_{j}}{r_{i j}}+A_{i j} \exp \left(-b_{i j} r_{i j}\right)-\frac{c_{i j}}{r_{i j}^{6}},
$$

where $q$ is an atomic charge and $A, b$ and $c$ are constants specified by the types of atoms $i$ and $j$. The interatomic force is then calculated from

$$
\mathbf{F}_{i j}=-\frac{\partial U_{i j}}{\partial \mathbf{r}_{i j}}
$$

The BKS potential has been used to predict the thermal conductivity of amorphous silica with reasonable accuracy ${ }^{22}$, and in a more limited case, the thermal conductivity of quartz and the siliceous forms of FAU and $\mathrm{LTA}^{21}$. Parameters are also available for the inclusion of aluminum, potassium and sodium in the simulation cell ${ }^{28,29}$.

The electrostatic interactions are modeled using the Wolf method ${ }^{30}$, where the first term in Eq. (12) is calculated as

$$
\frac{q_{i} q_{j}}{r_{i j}} \simeq \frac{q_{i} q_{j} \operatorname{erfc}\left(\alpha r_{i j}\right)}{r_{i j}}
$$




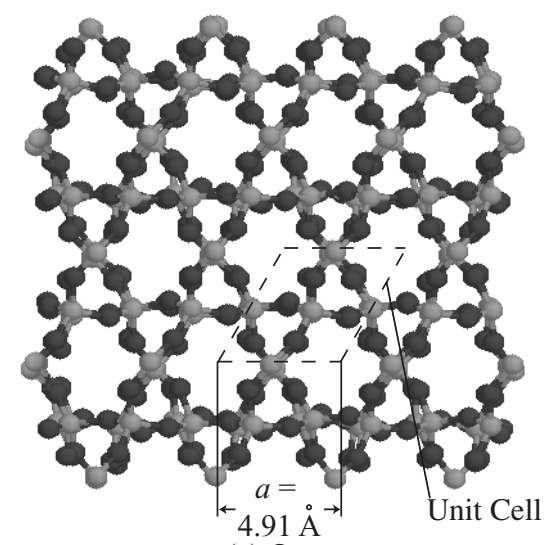

(a) Quartz

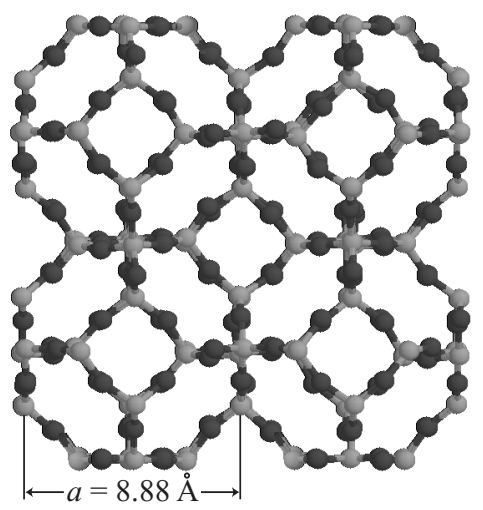

(b) Sodalite (SOD)

Figure 1: Simulation cells for (a) quartz and (b) sodalite. For quartz, the $c$-direction is perpendicular to the plane of the figure.

Using the Wolf method instead of the traditional Ewald sum is advantageous in that it significantly reduces the required computational time. The Wolf method is essentially equivalent to ignoring the long range electrostatic interactions. When the full Ewald sum was used in the current simulations, the long range force and energy contributions were on the order of one percent of the short range terms. This type of behavior has been reported previously ${ }^{31,32}$.

The parameter $\alpha$ in Eq. (14) provides the damping necessary to make the electrostatic interaction short range, and must be specified. Demontis et al. ${ }^{33}$ suggest choosing a value of $4 / L$, where $L$ is the size of the simulation cell. They show that for a range of crystals, including some zeolites, this value gives the closest agreement with the full Ewald sum. For the current simulations, a constant value of $\alpha$ is used so that the potential is the same for the different crystals studied. A value of $0.223 \AA^{-1}$ is chosen, which corresponds to a typical $4 / L$ for the SOD structure.

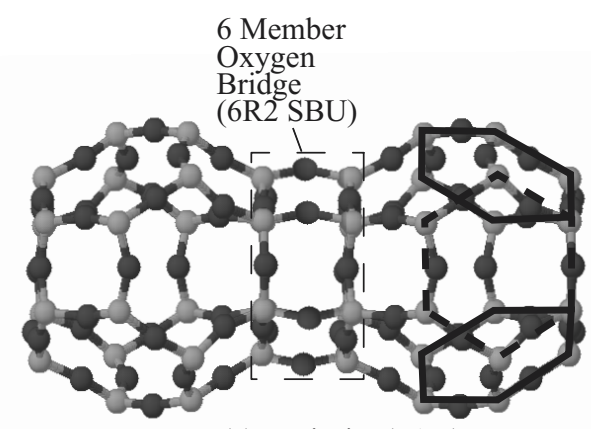

(a) Faujasite (FAU)

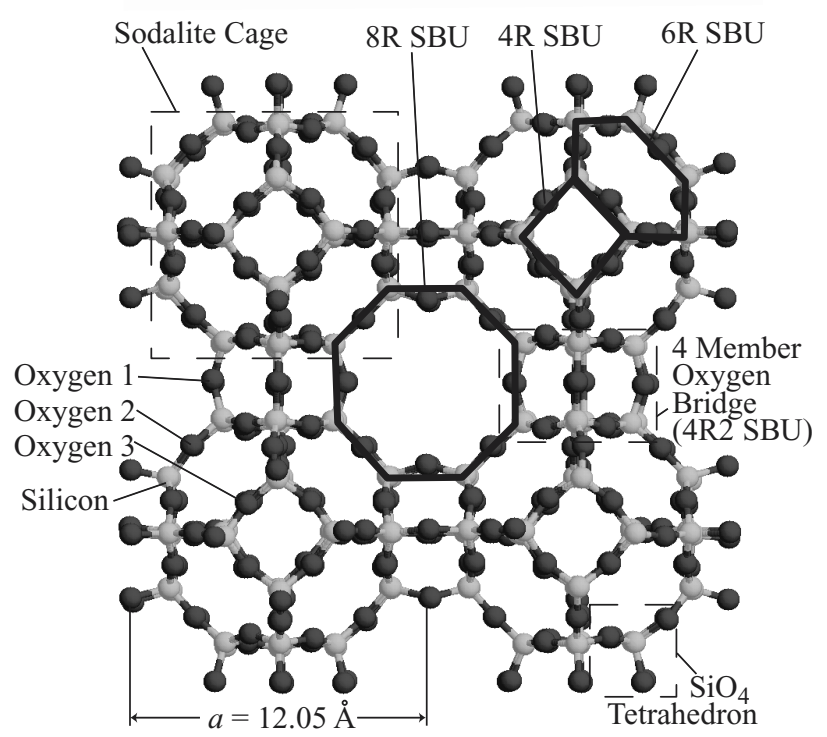

(b) Zeolite A (LTA)

Figure 2: (a) A portion of the faujasite simulation cell. Two sodalite cages are shown, joined by a six member oxygen bridge The connection points of the other cages on the right cage are indicated with bold lines. The dashed line indicates that the connection point is in the rear of the cage. The FAU structure also contains a 12R SBU. (b) The simulation cell for zeolite-A.

\section{Simulation Procedure}

All reported data corresponds to simulations in the $N V E$ (constant mass, volume and energy), or micro-canonical, ensemble. The time step used is 0.9 fs. The equations of motion are integrated with a Verlet leap-frog algorithm. The temperatures considered are between 100 and $350 \mathrm{~K}$ and the simulations are run at zero pressure. The interactions are truncated and shifted at a radius $R_{c}$ that is one half of the shortest side length of the simulation cell. Specifics of the simulations for each of the four crystals are given in Table 1 . In the table, $N$ is the number of atoms in the simulation cell and $\rho$ is density.

To determine the zero pressure cell size, simulations were run in the NPT (constant mass, pressure

4

American Institute of Aeronautics and Astronautics 
Table 1: MD simulation parameters. Dimensions and density are at $300 \mathrm{~K}$.

\begin{tabular}{ccccc}
\hline Structure & $\begin{array}{c}\text { Unit Cell, } \\
\AA\end{array}$ & $\begin{array}{c}\text { Atoms/ } \\
\text { Unit Cell }\end{array}$ & $N$ & $\begin{array}{c}\rho, \\
\mathrm{kg} / \mathrm{m}^{3}\end{array}$ \\
\hline $\mathrm{Q}$ & $4.91(a)$ & 9 & 576 & 2589 \\
& $5.52(c)$ & & & \\
SOD & 8.88 & 36 & 288 & 1705 \\
FAU & 24.72 & 576 & 576 & 1266 \\
LTA & 12.05 & 72 & 576 & 1368 \\
\hline
\end{tabular}

and temperature) ensemble in increments of $50 \mathrm{~K}$ between 100 and $350 \mathrm{~K}$. The temperature and pressure were controlled using a Nose-Hoover thermostat and barostat. To account for the cutoff of the potential, the pressure was corrected by ${ }^{34}$

$$
P_{\text {tail }}=\frac{2 \pi}{3}\left(\frac{N}{V}\right)^{2} \int_{R_{c}}^{\infty} r^{3} F(r) d r,
$$

which assumes that the local density beyond $R_{c}$ is uniform (i.e., the radial distribution function is equal to unity). For the quartz structure, the pressure was independently controlled in the $x, y$ and $z$ directions to allow for the proper relaxation of the non-cubic unit cell. For the zeolites, the ratio of the three lengths was fixed at unity. After an initialization period of $2 \times 10^{5}$ times steps ( $\left.0.18 \mathrm{~ns}\right)$, data was collected and averaged over $4 \times 10^{5}$ time steps $(0.36$ ns). The simulation cell size was determined at each temperature, and a second order polynomial was fit to the data. The resulting curve is used to specify the cell size in all subsequent simulations. Consistent with previous experimental and numerical work, LTA and FAU were found to contract with increasing temperature $^{21}$, while quartz and SOD were found to expand. The size of the unit cell for each structure at $300 \mathrm{~K}$ is given in Table 1 . Using the results of these simulations, the temperature dependence of the system potential energy was found. This result will be used in the temperature setting procedure.

For all subsequent simulations discussed, an initialization period of $5 \times 10^{5}$ time steps $(0.45 \mathrm{~ns})$ has been used. The system is run in the NVT (constant mass, volume and temperature), or canonical, ensemble for $3 \times 10^{5}$ time steps. The potential energy of the system is then monitored every time step. When it reaches a value within $10^{-4} \%$ of the fit potential energy-temperature function, the ensemble is switched to $N V E$, and the system is run until the total number of time steps is $5 \times 10^{5}$. This procedure allows the temperature to be set within about $1 \mathrm{~K}$ of the desired value. The number of time steps required to get a potential energy within this tolerance is typically less than 1000 . This method is advantageous in that it is passive, as compared to brute-force temperature scaling procedures. While running in the $N V E$ ensemble, the total energy is conserved to with $0.005 \%$.

For the structural data reported, a further $10^{5}$ time steps (90 ps) are run beyond the initialization period. The atomic coordinates are extracted at three instants in time over this period, and the relevant parameters (i.e. bond lengths and angles) are calculated and averaged over the structure and over time.

All simulations used in thermal conductivity calculations consist of an additional $10^{6}$ time steps (0.9 ns) over which the heat current is calculated every 5 time steps. A correlation length of 50,000 time steps (45 ps) with $2 \times 10^{5}$ time origins is used to extract the value of the thermal conductivity.

The specification of the integral in Eq.(8) is not trivial. In theory, the HCACF should decay to zero. This is not found. Instead, noise is present in the long time behavior. In some cases, this noise makes it difficult to determine the converged value of the integral. In some materials, such as $\beta$-silicon carbide ${ }^{12}$ and $\operatorname{argon}^{15}$, the HCACF decays essentially monotonically. Oscillations are small compared to the initial value, and the HCACF can be fit with an exponential function, allowing for a direct specification of the integral. In other cases, such as the current silica structures, the oscillations in the heat current are large, and an exponential fit is not suitable. This type of decay has been attributed to relative oscillations between bonded atoms with different masses ${ }^{13}$.

We propose and implement the following scheme. For each thermal conductivity value reported, five simulations were performed with different initial conditions. The resulting HCACFs were averaged, and the resulting function was integrated using the trapezoidal rule. The HCACF and its integral for the $a$ direction of quartz at $250 \mathrm{~K}$ are shown in Fig. 3. The oscillations in the integral are large, but it does converge. To establish where the convergence occurs, the integral is average in overlapping blocks of 2500 time steps. This curve is also shown in Fig. 3(b). A region of at least 5000 time steps is chosen over which the integral is deemed to have converged. The value of the integral is averaged over this region, and that is the value used to determine the thermal conductivity. This method was found to give better results that specifying the integral for each individual HCACF, and then averaging the resulting thermal conductivity values.

In some cases, the above method was not vi-

5

American Institute of Aeronautics and Astronautics 


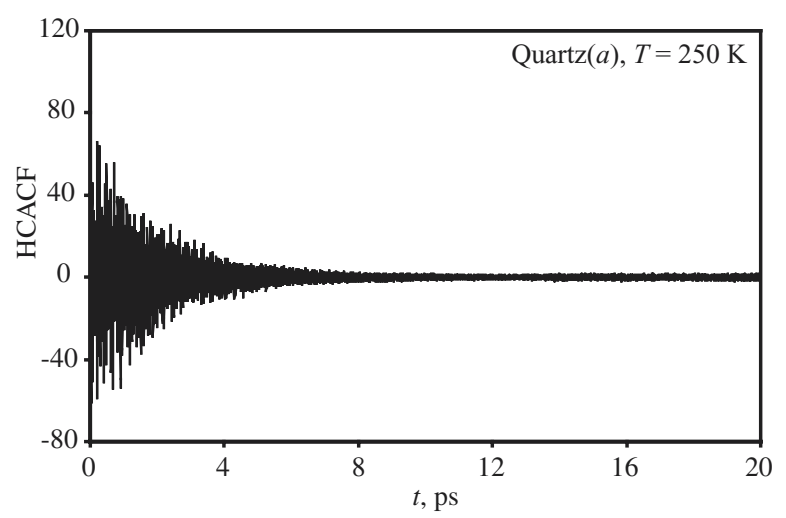

(a)

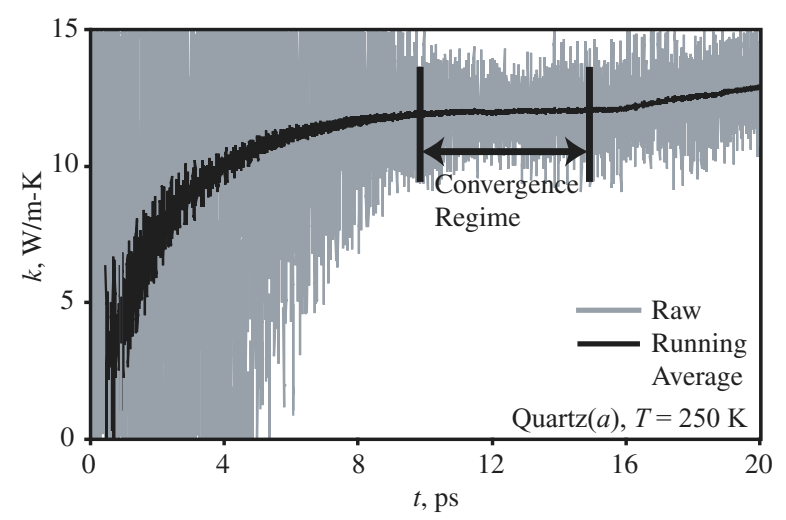

(b)

Figure 3: The (a)HCACF and (b)its integral (the thermal conductivity) for quartz $(a)$ at $250 \mathrm{~K}$. This is an example of a well converged value of the thermal conductivity. The integral is averaged over the indicated region.

able. As mentioned previously, noise in the HCACF can cause the integral to diverge. An example of this behavior is shown for the $c$-direction of quartz at $200 \mathrm{~K}$ in Fig. 4. To deal with these conditions, we note that the oscillations in the integral always reach a minimum (i.e., the integral necks) before the integral starts to diverge. This is shown in Fig. 4(b). By comparing to the cases where the integral clearly converges [e.g, Fig. 3(b)], it is found that the $\mathrm{HCACF}$ function beyond this point does not make a significant contribution to the integral. An average of the integral is taken over 1000 time steps around the neck, and this is the value used in the calculation of the thermal conductivity. The consistency in the calculated thermal conductivity values gives support to this method.

\section{RESULTS AND DisCUSSION}

\section{Thermal Conductivity}

A plot of the calculated thermal conductivities for the four crystals as a function of temperature is

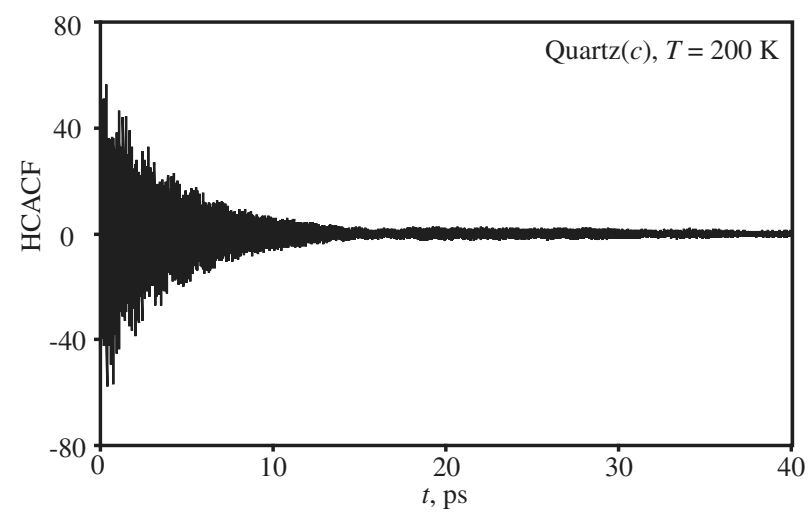

(a)

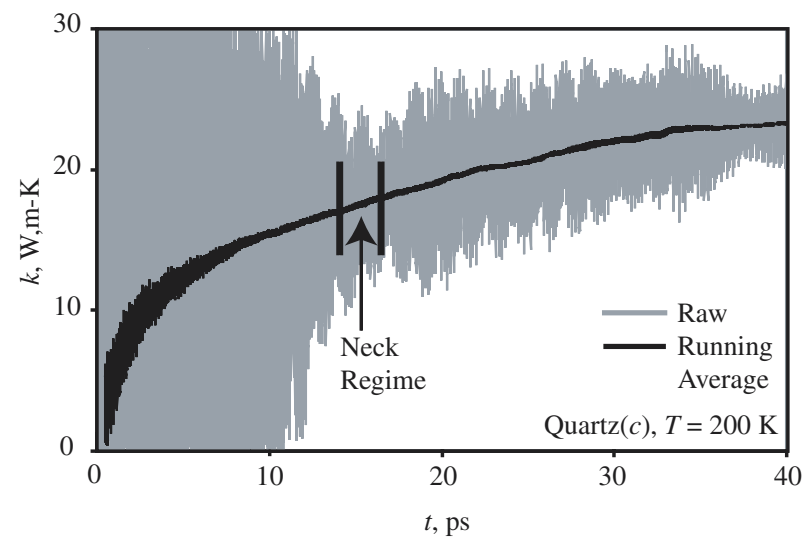

(b)

Figure 4: The (a)HCACF and (b) its integral (the thermal conductivity) for quartz(c) at $200 \mathrm{~K}$. This is an example of an integral that does not converge. The value of the integral is taken in the indicated neck regime.

shown in Fig. 5. The values are also presented in Table 2. Also included in the figure are experimental results for quartz and amorphous silica ${ }^{35}$, and the predictions of the high-scatter limit, Eq. (6). The amorphous silica parameters are taken from Cahill et al. ${ }^{8}$ For quartz, which is anisotropic, only the thermal conductivity in the $c$-direction is shown in the figure. The $a$-direction data are included in Table 2 . The error bars are an indication of the error in specifying the integral in Eq. (8). This is taken as $10 \%$ of the calculated value. Least squares power law fits are added for the three zeolites.

There is very little experimental or computational data available for zeolite thermal conductivity. Murashov $^{21}$ investigated LTA and FAU using MD, but appears not have run enough tests to get statistically meaningful results. The data are included in Table 2. The current MD calculations for LTA are in good agreement with Murashov's results, while the FAU values are higher. Griesinger et al. ${ }^{36}$ have measured the effective thermal conductivity of ze-

6

American Institute of Aeronautics and Astronautics 


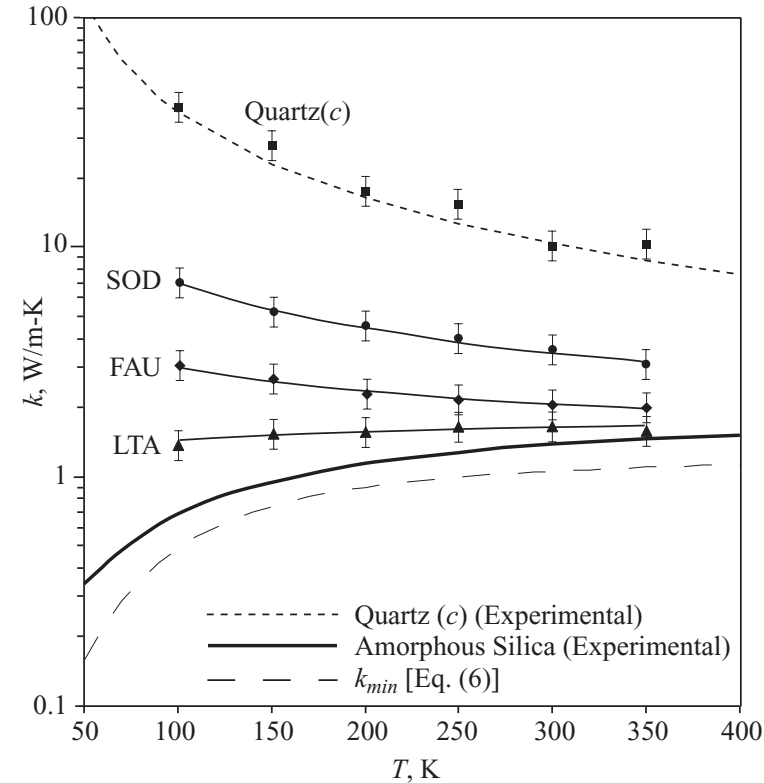

Figure 5: Predicted, experimental and theoretical thermal conductivities.

olite powders with various filling gases. They also present a network model for the prediction of the thermal conductivity that shows reasonable agreement with the experimental data. We note that the current work is concerned with bulk properties, which can be difficult to extract from powder data.

The experimental and MD results for the $c$-direction of quartz show agreement to within an average of $10 \%$. In the $a$-direction, the MD results are on average $40 \%$ higher than the experimental data. Based on other MD work that has used a Green-Kubo approach to calculate thermal conductivity ${ }^{12,18}$, this agreement is good. This gives confidence to the ability of the BKS potential to model thermal transport in silica structures. None of the crystals has a conductivity below the amorphous value, although the LTA curve is approaching the amorphous curve as the temperature increases. The high scatter limit falls below all of the experimental and MD results.

Of the simulated structures, quartz has the highest conductivity, followed by SOD, FAU and LTA. The general trend of the calculated conductivities can be thought of in terms of the overall stiffness of each of the structures. With its high density, quartz will be the stiffest. For the zeolites, consideration of the joining mechanisms between the sodalite cages suggests that SOD (where the cages are directly attached) will be the stiffest, followed by FAU (where the oxygen bridges contain six elements), followed by LTA (where the oxygen bridges contain four elements). This is consistent with the thermal conduc-
Table 2: The predicted thermal conductivities from the MD simulations. The numbers in square brackets under the quartz data correspond to experimental values from Ref. 35. The numbers in square brackets under the FAU and LTA data are the MD results from Ref. 21. Those simulations also predict quartz thermal conductivities of $16 / 8.2 \mathrm{~W} / \mathrm{m}-\mathrm{K}$ at $300 \mathrm{~K}$.

\begin{tabular}{ccccc}
\hline $\begin{array}{c}\text { Structure } \\
T, \mathrm{~K}\end{array}$ & $\mathrm{Q}(c / a)$ & $\begin{array}{c}\text { SOD } \\
k, \mathrm{~W} / \mathrm{m}-\mathrm{K}\end{array}$ & FAU & LTA \\
\hline 100 & $40.7 / 26.2$ & 7.1 & 3.0 & 1.4 \\
& {$[39 / 20.8]$} & & {$[1.4]$} & {$[1.2]$} \\
150 & $27.4 / 17.2$ & 5.3 & 2.7 & 1.6 \\
& {$[23.1 / 13]$} & & & \\
200 & $17.4 / 14.2$ & 4.7 & 2.3 & 1.6 \\
& {$[16.4 / 9.5]$} & & {$[1.3]$} & {$[1.5]$} \\
250 & $15.5 / 12.0$ & 4.0 & 2.2 & 1.7 \\
& {$[12.7 / 7.5]$} & & & \\
300 & $10.3 / 8.2$ & 3.6 & 2.1 & 1.7 \\
& {$[10.4 / 6.2]$} & & {$[0.6]$} & {$[1.3]$} \\
350 & $10.3 / 8.5$ & 3.1 & 2.0 & 1.6 \\
& {$[8.8 / 5.3]$} & & & \\
\hline
\end{tabular}

tivity trend.

Of interest are the trends within each structure and between the structures. Both directions for quartz, SOD and FAU show the expected decrease in the thermal conductivity above one-tenth of the Debye temperature ${ }^{37}$, which for quartz is 290 $\mathrm{K}^{2}$. We assume that the zeolites have a comparable value. The exponent of the power law fit decreases between the structures in the direction of decreasing conductivity. For LTA, all of the calculated thermal conductivities are within a range of $0.3 \mathrm{~W} / \mathrm{m}-\mathrm{K}$, which approaches the resolution of the predictions. A slight increase in the conductivity over the temperature range considered is discernable.

A possible interpretation of this result is that LTA is displaying the thermal conductivity trend of an amorphous solid or disordered crystal, as predicted by Eq. (6). A maximum phonon scattering (or complete localization) condition has been reached. The decrease in the exponent of the power law fits in the other structures is consistent with a transition from the crystalline behavior to the amorphous behavior.

In the low temperature limit, experimental measurements of crystalline thermal conductivity reach a maximum, and then decrease to zero. At low temperatures, phonon scattering is dominated by impurities and boundary effects. These scattering mechanisms are not present in the current simulations, 


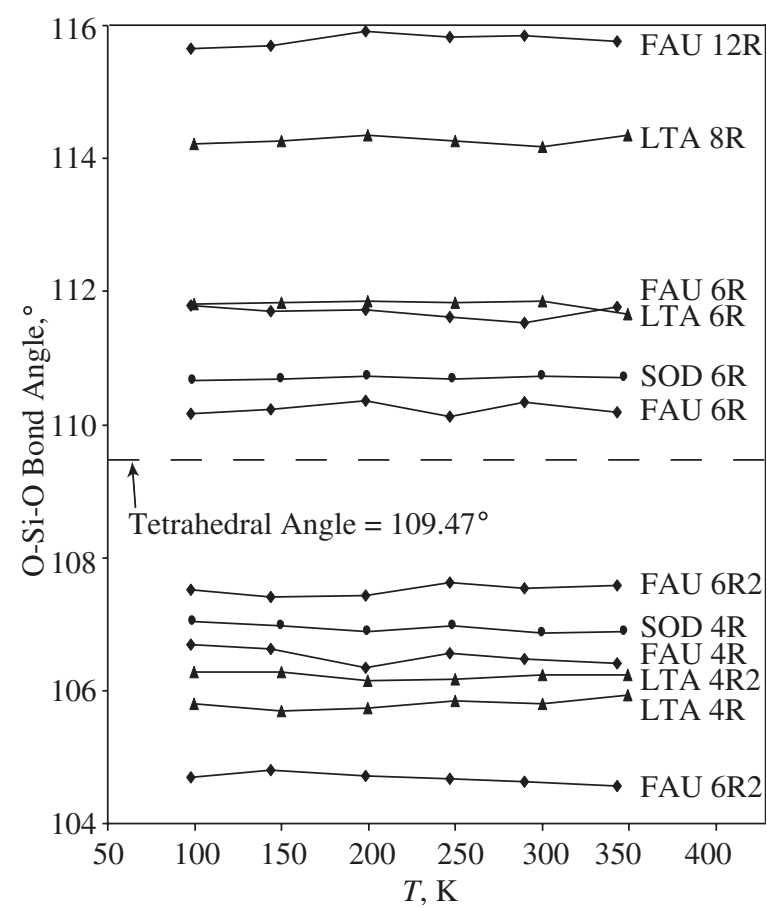

Figure 6: The tetrahedral angles found using MD in the zeolites. The angles can be grouped based on the SBU with which they are associated.

and it would be expected that the thermal conductivities will continue to increase as the temperature is decreased due to the increasingly harmonic nature of the atomic interactions. Also, at lower temperatures, quantum effects will become important, and it would not be expected that the MD calculations will be in as good agreement with the experimental data.

In the next two sections, we search for evidence of phonon scattering in the lattice statics and dynamics, and for indications of what about the structures of the different crystals leads to their different thermal conductivities.

\section{LATTice Structure}

The thermal conductivity trends can be seen in the simulations by considering the root mean square (RMS) displacement of the atoms or the standard deviation of the bond length and angle distributions. A higher RMS or standard deviation will correspond to larger motions away from the equilibrium positions, and more anharmonic interactions. This trend is observed for increasing temperature for all the structures considered, and is consistent with the decreasing thermal conductivity found for quartz, SOD and FAU. In LTA, there is no effect on the thermal conductivity because the high scatter limit has been
Table 3: Comparison of the distortion of the tetrahedra in the four structures and their thermal conductivities at $300 \mathrm{~K}$.

\begin{tabular}{cccc}
\hline Structure & $\begin{array}{c}\text { O-Si-O, } \\
\text { Angles, }\end{array}$ & $\begin{array}{c}\text { Mean Dev. } \\
\text { From Tetra. } \\
\text { Angle, }\end{array}$ & $\begin{array}{c}k, \\
\mathrm{~W} / \mathrm{m}-\mathrm{K}\end{array}$ \\
\hline SOD & 4 @ 110.7 & 1.68 & 3.6 \\
& 2 @ 106.9 & & \\
FAU & 1 @ 115.8 & 3.16 & 2.1 \\
& 1 @ 111.7 & & \\
& 1 @ 110.2 & & \\
& 1 @ 107.5 & & \\
& 1 @ 106.5 & & \\
LTA & 1 @ 104.7 & & \\
& 1 @ 114.3 & 3.28 & \\
& 2 @ 111.8 & & \\
& 2 @ 106.2 & & \\
& 1 @ 105.8 & & \\
\hline
\end{tabular}

reached.

While the zeolites considered are distinguishable based on their unit cells, they can also be characterized by the local environment of each Si atom. As mentioned, the cages in the zeolites distort the positions of the oxygen atoms away from the perfect tetrahedral positions. This can be seen in Fig. 6 , where the variation of the $\mathrm{O}-\mathrm{Si}-\mathrm{O}$ bond angles in each of the zeolites (as calculated from the MD simulations) is plotted as a function of temperature. The zeolites can be compared as they are all constructed from sodalite cages using different joining mechanisms. As quartz does not contain SBUs or sodalite cages, its inclusion in this analysis is not appropriate.

From Fig. 6, the resulting angles for the three zeolites can be organized based on their associated SBU. The largest angles are those associated with the large inter-cage pores, followed by those in the $6 \mathrm{R}$ SBUs. Below the tetrahedral angle, the angles associated with the $4 \mathrm{R}$ SBU and oxygen bridges are found. In Table 3, the mean deviation of the angles in a tetrahedron for each of the zeolites is calculated based on the average bond angle over the temperature range considered. As the deviation increases, the thermal conductivity decreases. Thus, the distortion of the small scale structure can be associated with an increase in phonon scattering.

Murashov $^{21}$ has investigated the thermal conductivity of LTA when some of the silicon atoms are replaced with other species. These replacements lead to a distortion of the tetrahedra (which can also be shown using radial distribution functions) and an

8

American Institute of Aeronautics and Astronautics 
accompanying decrease in the thermal conductivity. Here, we see that the crystal structure itself can have the same effect.

\section{VibRATIONAL SPECTRA}

Phonon relaxation times can be extracted from the imaginary part of the infrared spectrum ${ }^{38}$. The width of a peak in the spectrum is related to the reciprocal of the phonon relaxation time for that mode. Using experimental spectra and some fitting parameters, Hofmeister ${ }^{38}$ has used this concept to develop a thermal conductivity model for various materials found in the earth's mantle.

A significant amount of work has been done to relate the spectra of zeolites to their structural features $^{39}$. While some general guidelines have been suggested, no definitive interpretations of the spectra have been established.

Using MD, the infrared spectrum can be found by taking the Fourier transform of the dipole moment autocorrelation function ${ }^{11}$. We find the location of the spectral peaks of the Fourier transform of the HCACF to be consistent with those of the dipole moment. This is likely due to the strong contribution of the electrostatic term in the force and energy calculations in Eq. (10). As the HCACF is what is used to determine the thermal conductivity, its spectrum will be considered in the ensuing analysis.

The power spectra of the HCACF for all the structures at $300 \mathrm{~K}$ are shown in Fig. 7. The spectra show qualitative agreement with the infrared spectra found for zeolites in previous MD simulations ${ }^{40,41}$. There are some discrepancies in the peak heights and locations. This may be due to the consideration of the heat current as opposed to the dipole moment, and the different interatomic potentials used.

While the location of the peaks is independent of the length of the time sample used in the Fourier transform, the width of the peaks is affected. As the length of the time sample increases, the peaks get narrower. The advantage of using a longer time sample is that the peaks are better defined, and it is possible to resolve more features. All of the spectra shown in Fig. 7 were formed using the entire 50,000 time steps in the HCACF. We are thus able to compare how the spectra of a given structure change with temperature, but comparisons between structures and quantitative analysis are difficult. A plot of the phonon relaxation times associated with the quartz $a$-direction is shown in Fig. 8. As the temperature increases, the relaxation times decrease. The resulting decrease in the thermal conductivity is consistent with Eq. (3). The phonon relaxation times are consistent with the decay times of the HCACF

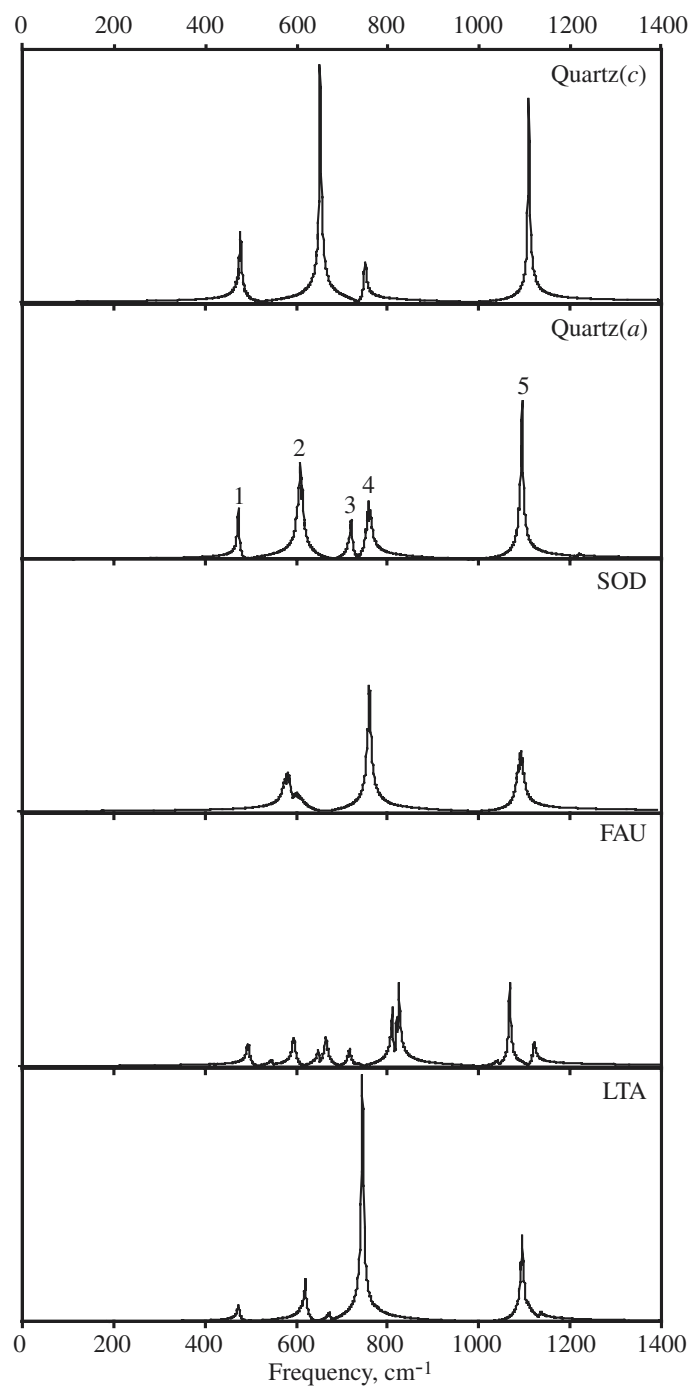

Figure 7: The power spectra of the HCACF for the four structures (including both the $a$ - and $c$-directions for quartz). The vertical scale is the same for all five plots.

[see Figs. 3(a) and 4(a)].

The integration in Eq. (8) can be performed over frequency as opposed to over time. By taking the Fourier transform of the HCACF, filtering out all frequencies beyond a value $\omega$, taking the inverse Fourier transform and then integrating up to the convergence time period identified in the initial time analysis, the progression of the integral in frequency space can be studied. A sample of the resulting curves for SOD are shown in Fig. 9 along with the spectrum of the $\mathrm{HCACF}$ at $250 \mathrm{~K}$.

To understand the curves in Fig. 9, a mathematical model of the HCACF can be constructed. Suppose that the HCACF can be expressed as the 


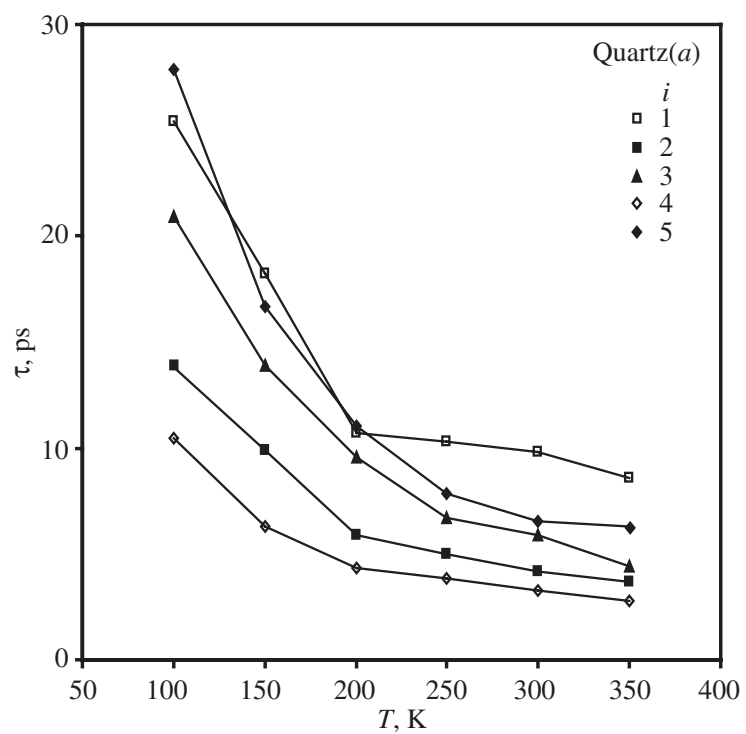

Figure 8: Predicted phonon relaxation times for the peaks in the HCACF of quartz $(a)$. The peak numbers refer to those defined in Fig. 7.

sum of a number of exponentially decaying sinusoids:

$$
\langle\mathbf{q}(t) \cdot \mathbf{q}(0)\rangle=\sum_{i} A_{i} \exp \left(-t / \tau_{i}\right) \cos \left(\omega_{i} t\right) .
$$

where $A, \tau$, and $\omega$ are constants. The summation is over the $i$ peaks of the spectrum. There are two important time parameters associated with each term: $\tau$ is the time constant for the decay of the exponential envelope, and $\omega$ is the frequency of the oscillations. The Fourier transform of each term in Eq. (16) is a single peak whose height, width and location are related to $A, \tau$ and $\omega$ respectively. Based on Eqs. (8) and (16), the thermal conductivity will be given by

$$
k=\frac{1}{3 k_{B} V T^{2}} \sum_{i} \frac{A_{i} \tau_{i}}{1+\tau_{i}^{2} \omega_{i}^{2}} .
$$

Note that all the terms in the summation are positive. While the integration generates negative values at low frequencies, the only significant value of the conductivity is the final, asymptotic limit (similar to the interpretation in the time domain). This result can be interpreted as finding the thermal conductivity by summing over the contributions of the distinct frequencies found in the system, which is analogous to Eq. (3). Large, narrow peaks will make a larger contribution than small, broad peaks. Thus, the damping of specific peaks will result in a shorter relaxation time, and a decrease of the thermal conductivity.

An ideal method for specifying the integral in Eq.(8) would be to fit the HCACF with a function

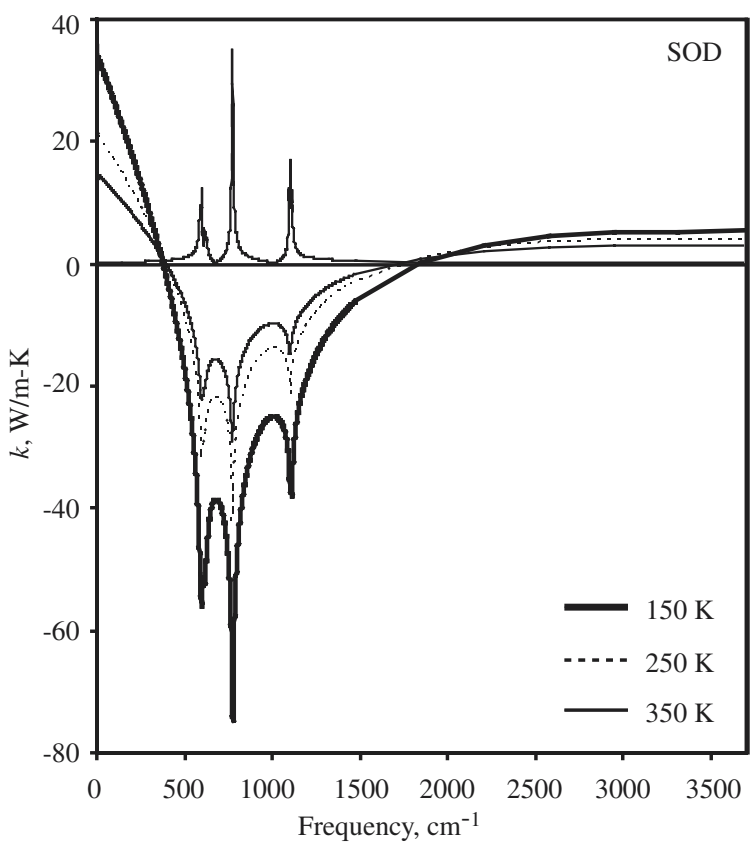

Figure 9: The HCACF for sodalite integrated over frequency. The power spectrum at $250 \mathrm{~K}$ is also included.

like Eq.(16) and use the result of Eq.(17). In practice, this is difficult to do for two reasons. First, as seen in Fig. 7, some of the spectra contain many peaks, some of which are close together. The mathematical task of fitting a function with up to 30 unknowns is formidable. Secondly, if the real form of the HCACF were Eq.(16), then the noise observed in the MD results would not be present.

The frequency space analysis reveals that the source of the noise is the very low frequency modes of the spectra. Filtering out of all frequencies below around $25 \mathrm{~cm}^{-1}$ results in an integral with definite convergence. However, filtering out these frequencies will have a significant effect on the value of the integral, as can be seen in Fig. 9. Attempts to smooth out the low frequency behavior by fitting it with low-order polynomials were unable to generate consistent results.

\section{Conclusion}

The MD simulations described in this study have demonstrated the suitability of the BKS interatomic potential for predicting the thermal conductivity of silica structures between temperatures of 100 and $350 \mathrm{~K}$. The thermal conductivity trends are consistent with qualitative and quantitative arguments based on the crystal structures and phonon scattering mechanisms. For zeolite-A, the conductivity is essentially constant over the temperature range considered. This is interpreted as the attainment of the 
high scattering limit generally associated with amorphous materials. The analysis of the lattice statics and dynamics suggests that the thermal conductivity of crystalline solids can be lowered through the introduction of small scale disorder, and by damping specific structural features.

\section{ACKNOWLEDGEMENTS}

This research has been sponsored by the U.S. Department of Energy, Office of Basic Energy Sciences under contract DE-FG02-00ER45851 and the Natural Sciences and Engineering Research Council of Canada (AJHM).

\section{REFERENCES}

1. M. Kaviany, Principles of Heat Transfer in Porous Media, Second Edition (Springer, New York, 1995).

2. M. Kaviany, Principles of Heat Transfer (Wiley, New York, 2001).

3. R. Berman, Thermal Conductivity of Solids (Clarendon, Oxford, 1976).

4. J. Callaway, Phys. Rev. 113, 1046 (1959).

5. M. G. Holland, Phys. Rev. 132, 2461 (1963).

6. D. P. White, J. Nucl. Mater. 212-215, 1069 (1994).

7. D. G. Cahill and R. O. Pohl, Solid State Commun. 70, 927 (1989).

8. D. G. Cahill, S. K. Watson and R. O. Pohl, Phys. Rev. B 46, 6131 (1992).

9. A. Einstein, Ann. Physics 35, 679 (1911).

10. G. A. Slack, in Solid State Physics, eds. F. Seitz and D. Turnbull (Academic Press, New York, 1979).

11. D. A. McQuarrie, Statistical Mechanics (University Science Books, Sausilito, 2000).

12. J. Li, L. Porter and S. Yip, J. Nucl. Mater. 255, 139 (1998)

13. J. Che, T. Cagin, W. Deng and W. A. Goddard III, J. Chem. Phys. 113, 6888 (2000).

14. K. Takese, I. Akiyama and N. Ohtori, Mater. T. JIM 40, 1258 (1999).

15. H. Kaburaki, J. Li and S. Yip, Mat. Res. Soc. Symp. Proc. 538, 503 (1998).

16. P. Keblinski, S. R. Phillpot, S. U. S. Choi and J. A. Eastman, Int. J. Heat Mass Tran. 45, 855 (2002).

17. Y. H. Lee, R. Biswas, C. M. Soukoulis, C. Z. Wang, C. T. Chan and K. M. Ho, Phys. Rev. B 43, 6573 (1991).

18. S. G. Volz and G. Chen, Phys. Rev. B 61, 2651 (2000).

19. S. Motoyama, Y. Ichikawa, Y. Hiwatari and A. Oe, Phys. Rev. B 60, 292 (1991).
20. R. Inoue, H. Tanaka and K. Nakanishi, J. Chem. Phys. 104, 9569 (1996).

21. V. V. Murashov, J. Phys-Conden. Mat. 11, 1261 (1999).

22. P. Jund and R. Jullien, Phys. Rev. B 59, 13707 (1999).

23. J. R. Lukes, D. Y. Li, X.-G. Liang and C.-L. Tien, J. Heat Transf. 122, 536 (2000).

24. F. Muller-Plathe, J. Chem. Phys. 106, 6082 (1997).

25. D. Bedrov and G. D. Smith, J. Chem. Phys. 113, 8080 (2000).

26. A. Dyer, An Introduction to Zeolite Molecular Sieves (Wiley, Chichester, 1988).

27. R. W. G. Wyckoff, Crystal Structures, Volume 4 (Interscience, New York, 1968).

28. B. W. H. van Beest, G. J. Kramer and R. A. van Santen, Phys. Rev. Lett. 62, 1955 (1990).

29. G. J. Kramer, N. P Farragher, B. W. H. van Beest and R. A. van Santen, Phys. Rev. B 43, 50 (1991).

30. D. Wolf, P. Keblinski, S. R. Phillpot and J. Eggebrecht, J. Chem. Phys. 110, 8254 (1999).

31. Z. A. Rycerz and P. W. M Jacobs, Mol. Simulat. 8, 197 (1992).

32. Z. A. Rycerz, Mol. Simulat. 9, 327 (1992).

33. P. Demontis, S. Spann and G. B. Suffritti, J. Chem. Phys. 114, 7980 (2001).

34. D. Frenkel and B. Smit, Understanding Molecular Simulation: From Algorithms to Applications (Academic Press, San Diego, 1996).

35. Y. Touloukian, Thermophysical Properties of Matter Volume 2 (Plenum, New York, 1970).

36. A. Griesinger, K. Spindlet and E. Hahne, Int. J. Heat Mass Tran. 42, 4363 (1999).

37. A. Majumdar, in Microscale Energy Transport, eds. C.-L. Tien, A. Majumdar and F. G. Gerner (Taylor and Francis, Washington, 1998).

38. A. M. Hofmeister, Science 283, 1699 (1999).

39. A. J. M. de Man and R. A. van Santen, Zeolites 12, 269 (1992).

40. P. Bornhausen and D. Bougeard, J. Phys. Chem. $B$ 105, 36 (2001).

41. K. S. Smirnov and D. Bougeard, Catal. Today 70, 243 (2001). 